NASA Technical Memorandum 83587

\title{
A Theoretical Prediction of the Acoustic Pressure Generated by Turbulence- Flame Front Interactions
}

Ronald G. Huff

Lewis Research Center

Cleveland, Ohio

Prepared for the

Winter Annual Meeting of the American Society of Mechanical Engineers

New Orleans, Louisiana, December 9-13, 1984 


\section{A THEORETICAL PREDICTION OF THE ACOUSTIC PRESSURE GENERATED BY TURBULENCE-FLAME FRONT INTERACTIONS}

Ronald G. Huff

National Aeronautics and Space Administration

Lewis Research Center

Cleveland, Ohio 44135

ABSTRACT

The equations of momentum and continuity are combined and linearized yielding the one dimensiona? nonhomogeneous acoustic wave equation. Three terms in the nonhomogeneous equation act as acoustic sources and are taken to be forcing functions acting on the homogeneous wave equation. The three source terms are: fluctuating entropy, turbulence gradients, and turbulence-flame interactions. Each source term is discussed. The turbulence-flame interaction source is used as the basis for computing the source acoustic pressure from the Fourier transformed wave equation. Pressure fluctuations created in turbopump gas generators and turbines may act as a forcing function for turbine and propellant tube vibrations in earth to orbit space propulsion systems and could reduce their life expectancy. A preliminary assessment of the acoustic pressure fluctuations in such systems is presented.

\section{INTRODUCTION}

Acoustic pressure fluctuations created in flowing systems may be the source of unwanted noise and serve as the unsteady driving force acting on mechanical systems associated with the flow. Aircraft type turbine engine noise has been a problem that has received attention over the past several years. High bypass turbofan engines with lower jet velocities have resulted in large reductions of aircraft jet noise. With the reduction of jet noise, other noise sources have emerged presenting additional problems for researchers working in the noise reduction field. The combustor is one of the acoustic sources whose noise characteristics are shown to be discernible in the farfield under some circumstances (1-2). Aircraft engines operating at reduced power settings have been shown to emit low frequency acoustic waves from the combustor that are transmitted through the turbine, ducting and nozzle to the farfield (3-4). Combustor noise may be expected from any turbuTent combustion process.

Of particular timely interest is the similarity between components used in aircraft turbine engine combustor-turbine configurations and the turbopump preburner-turbine configurations used to pump propellants in earth to orbit propulsion systems (5). Two of the primary differences are the propellants and method of mixing the propellants prior to combustion. In both systems, however, after the fuel is mixed with the oxidizer, the combustion process proceeds, the resulting gas expands through the turbine and then flows through the remaining ducting to either the core engine nozzle or to the main rocket engine dome where it passes over liquid oxygen ( $L O X$ ) tubes in semi-crossflow. For the aircraft engine the pressure fluctuations are the source of unwanted sound. For the space propulsion system the pressure fluctuations become an unsteady driving force causing vibrations in the LOX tubes that could be destructive if not accounted for in their design.

The combustor has been the subject of numerous theoretical and experimental investigations. Reference (6) discusses a few of the reported studies on combustion noise. The objective of the present paper is to present a simplified theoretical model, and prediction of the acoustic pressure generated in the combustor. Using this model a better understanding of the sound sources in combustors can be obtained enabling one to modify and predict their behavior. All symbols used in this paper are defined in the symbol list, Appendix A.

\section{COMBUSTION NOISE SOURCE MODEL}

The model for combustion noise considers onedimensional duct flow (Fig. 1). Fuel is sprayed into the airstream where turbulence generators, termed turbulators, promote mixing of the fuel with the air (oxidizer). A flame front perpendicular to the duct walls exists downstream of the turbulators at $x=$ 0 . The combustion process begins at the flame front and extends downstream to $X=L_{b}$ the point where burning ceases. The region between $X=0$ and $L_{b}$ is called the combustion zone. The duct extends to infinity in the $x$ direction. 
THE GOVERNING DIFFERENTIAL EQUATIONS AND ASSUMPTIONS

The second order partial differential equation governing the noise generated within an inviscid, adiabatic, perfect gas, in a one-dimensional duct with negligible fuel mass may be derived using the equations for conservation of mass and momentum respectively given here as:

$$
\begin{aligned}
& \frac{\partial \rho}{\partial t}=-\frac{\partial \rho V}{\partial x} \\
& \frac{\partial \rho V}{\partial t}=-\frac{\partial \rho V^{2}}{\partial x}-\frac{\partial P}{\partial x}
\end{aligned}
$$

\section{ACOUSTIC SOURCE AND WAVE EQUATIONS}

The acoustic wave equation describing the sound propagation in a duct is derived by first taking the partial derivative of Eq. (1) with respect to time; then taking the partial derivative of $\mathrm{Eq}$. (2) with respect to $x$, adding equations;

and then adding $\left(1 / C_{0}{ }^{2}\right)\left(\partial^{2} \mathrm{P} / \partial \mathrm{t}^{2}\right)$ to both sides of the resulting equation giving:

$$
\frac{1}{c_{0}^{2}} \frac{\partial^{2} p}{\partial t^{2}}-\frac{\partial^{2} p}{a x^{2}}=-\frac{\partial^{2} \rho}{\partial t^{2}}+\frac{1}{c_{0}^{2}} \frac{\partial^{2} p}{\partial t^{2}}+\frac{\partial^{2} \rho v^{2}}{\partial x^{2}}
$$

The assumption is made that the instantaneous pressure $P$, density $\rho$ and velocity $V$ are the sum of mean and fluctuating components, that is:

$$
\begin{aligned}
& P=P(x)+p(x, t) \\
& \rho=\rho(x)+\delta(x, t) \\
& V=V(x)+v(x, t)
\end{aligned}
$$

Substituting Eqs. (4) to (6) into Eq. (3), expanding and equating like order terms yields the wave equation as:

$\frac{1}{c_{0}{ }^{2}} \frac{\partial^{2} p}{\partial t^{2}}-\frac{\partial^{2} p}{\partial x^{2}}=\frac{\partial^{2}}{\partial t^{2}}\left(\frac{p}{c_{0}^{2}}-\delta\right)+2 \frac{\partial^{2} \rho v v}{\partial x^{2}}+\frac{\partial^{2} \delta v^{2}}{\partial x^{2}}$

To this point in the derivation nothing has been assumed concerning $C_{0}$ in the above equation. In this work, $\mathrm{C}_{0}$, is assumed to be the sonic velocity. Equation (7) then becomes the acoustic wave equation. It is assumed that outside the combustion zone the sonic velocity is constant, though different, in the upstream and downstream portions of the duct. It is further assumed that turbulence generated pressure fluctuations anywhere in the duct external to the combustion zone are less than the acoustic pressures generated by the combustion process and that no externally generated acoustic waves pass through the combustion zone.

The acoustic pressure is usually given in terms of finite-bandwidth, mean-square pressure fluctuations as a function of frequency. This is accomplished by taking the. Fourier transform of $\mathrm{Eq}$. (7), which removes time as a variable and thus simplifies the method of solution. The Fourier transform of Eq. (7) in terms of wave number $k$ is:

$$
\frac{\partial^{2} p_{\omega}}{\partial x^{2}}+k^{2} p_{\omega}=-\sum_{n=1}^{3} \varphi_{n, \omega}(x, \omega)
$$

where on are the Fourier transforms of the terms on the right side of Eq. (7). These terms are the acoustic sources, i.e. forcing functions, driving the acoustic wave equation. The source terms are of great interest since knowledge of their characteristics will give insight on how the fluctuating pressures found in the duct are generated. The primary thrust of this work is to define these source terms in detail. Before this can be accomplished, however, the nonhomogeneous equation (Eq. (8)) must be solved.

\section{SOLUTION OF NONHOMOGENOUS WAVE EQUATION}

By using the methods given in Ref. (8) the complete solution of the homogeneous part (i.e., with $\varphi=0$ ) of $\mathrm{Eq}$. (8) is

$$
p_{w, 0}=c_{1} e^{i k x}+c_{2} e^{-i k x}
$$

With the above complete solution known, the Green's function (9) may be used to write the equation for the fluctuating pressure in the duct propagating away from the combustion zone which constitutes the solution of the nonhomogeneous wave equation (Eq.

(8)). For the left and right running waves respectively the pressure is given by:

$$
\begin{aligned}
& p_{\omega, L}=\frac{e^{i k x}}{2 i k} \int_{0}^{L_{b}} \varphi_{\omega}(\xi) e^{-i k \xi} d \xi \\
& p_{\omega, R}=\frac{e^{-i k x}}{2 i k} \int_{0}^{L_{b}} \varphi_{\omega}(\xi) e^{i k \xi} d \xi
\end{aligned}
$$

where

$$
\varphi_{\omega}=-\left(\varphi_{\omega, 1}+\varphi_{\omega, 2}+\varphi_{\omega, 3}\right)
$$

The Fourier transformed source terms (i.e. Fourier transform of terms on the right side of Eq. (7)) are defined as:

$$
\varphi_{\omega, 1}=-\omega^{2}\left(\frac{p_{\omega}}{C_{0}^{2}}-\delta_{\omega}\right)
$$

or from Appendix B, Eq. (B14) in terms of fluctuating entropy

$$
\begin{aligned}
& \varphi_{1, \omega} \equiv-\frac{\omega^{2} \rho S_{\omega}^{\prime}}{C_{p}} \\
& \varphi_{2, \omega} \equiv \frac{\omega}{A} \frac{\partial^{2} v_{\omega}}{\partial X^{2}}
\end{aligned}
$$




$$
\varphi_{3, \omega} \equiv \frac{W}{A} \frac{\partial^{2}}{\partial x^{2}}\left(\frac{\delta \omega}{0} v\right)
$$

The source terms given by Eqs. (13) to (15) represent the acoustic sources due to: fluctuating heat release, turbulence, and energy addition (or withdrawal) respectively. Each has been developed in Appendix $B$ and will be discussed in greater detail below.

\section{PHYSICS OF NOISE GENERATION PROCESS}

The fluctuating entropy source term $\mathrm{Eq}$. (13) may be written from Appendix B, Eq. (B16) as:

$$
\varphi_{1, \omega}=-\frac{\omega^{2} \rho H_{v} f_{0, \omega}}{C_{p} T}
$$

Where the local fuel/air ratio, $f_{0}$, is allowed to fluctuate either because of unsteady turbulent mixing or because of some externally generated disturbance such as reflected acoustic waves passing through the fuel/air mixing region upstream of the flame front causing increased mixing. Thus this source may represent the energy addition due to acoustic feedback. As such one might expect this source to yield information on instability in combustors. In order to evaluate this source more information is needed on the local fuel/air ratio and the effect that turbulence and acoustic waves have on the mixing of the fuel with the air (oxidizer).

The second source term (Eq. (14)), describes the source of pressure fluctuations associated with the fluctuating velocity gradient. Thus the generation of fluctuating velocities by the turbulators or turbulent mixing of fuel and oxidizer in rocket engines is a source of noise in these flowing systems. This source term depends only on the mass flow per unit cross sectional area and the second derivative of the fluctuating velocity with respect to distance. (The second derivative with respect to distance is hereinafter called the Laplacian). Hence it is considered as the cold flow noise source that exists even if combustion is absent. This source term has been developed in Appendix $B$ for turbulence generated over a given length, LT. The turbulence at the end of the generator is assumed to have reached its maximum value and decay of the turbulence downstream of the generator is neglected. The second source term from Appendix B, Eq. (B20) is:

$$
\varphi_{2, \omega}=-2 \frac{W}{A} v_{w, L}\left(\lambda_{L_{T}}\right)^{2} e^{\frac{-\lambda}{L_{T}} X_{T}}
$$

The third acoustic source term 93, (Eq. (15)) is due to the Laplacian of the product of the turbulent density and the mean velocity. It has been assumed in this work that the turbulent density fluctuations in the combustion zone are constant. With this assumption this source term becomes a function of the Laplacian of the mean velocity, the result of the heat energy added by the combustion process. This source is investigated in detail in Ref. (6). The third source term from Appendix, B, Eq. (B2I). is expressed as:

$$
\varphi_{3, \omega}=-\frac{\omega^{\infty}}{A} \frac{\delta}{\rho} v_{0} \frac{{ }_{0}{ }_{C}{ }^{H} v^{f} 0_{0}{ }^{2}}{C_{p} T, 0} e^{-\lambda x_{b}}
$$

\section{ACOUSTIC SOURCE TERM EVALUATION}

In this paper no attempt will be made to evaluate the fluctuating entropy or cold flow acoustic sources $\varphi 1, \omega$ and $\varphi_{2} \omega^{\text {. Th }}$ The fluctuating entropy source $\phi_{1}, \omega$, should have low pressure levels if the burner ${ }^{\omega}$ operates stably. The cold flow noise term $\$ 2, \omega$ appears to act as an upstream fluctuating density generator and as such is amplified when passing through the combustion zone. Thus the third source term $\varphi_{3}$, w is expected to be the dominant one.

To evaluate $\mathrm{p}_{\omega, \mathrm{L}}$ (Eq. (10)), the source term $\$ 3, \omega$ must be given as a function of distance $x$. ${ }^{\prime}$ It is shown in Ref. (6) and Appendix $B$ that for Mach numbers much less than unity the velocity is directly proportional to the heat energy input to the air (Appendix B, Eq. (B22)).

By assuming that the fuel droplet mass decreases exponentially with axial distance, the velocity distribution due to the energy released to the air can be determined as a function of $x$. The second derivative with respect to $x$ is then substituted into $E q$. (15). The Fourier-transformed source term from Appendix $B$, (Eq. (21)) is

$$
\varphi_{3, \omega}=-\frac{\omega}{A} \frac{\delta_{\omega}}{\rho} v_{0} \frac{{ }_{{ }_{C}}{ }^{H} v^{f} 0^{\lambda}{ }^{2}}{C_{p} T_{T}, 0} e^{-\lambda x}
$$

Inserting Eq. (19) into Eq. (10), assuming that the fluctuating density is constant with distance through the combustion zone, and integrating yields the acoustic pressure in polar form as (see derivation in Appendix B, of Ref. ( 6$)$.

$$
p_{\omega, L}=\frac{{ }^{n} C}{2} \frac{\delta_{\omega}}{\rho} \frac{W}{A} \frac{V_{0} H_{0} f_{0}}{C_{p} T_{T, 0}} \frac{\lambda}{k} \frac{e^{i\left[k x+(\pi / 2)+\tan ^{-1}(-k / \lambda)\right]}}{\left[1+(k / \lambda)^{2}\right]^{1 / 2}}
$$

where $0<x<L_{b}$. Equation (20), with dimensions of pressure per unit angular frequency, is used for calculating the overall sound pressure level and the narrowband acoustic spectrum. The amplitude for the right and left running waves are calculated using. Eq. (20) with the appropriate sonic velocity inserted in the wave number, $k$.

In deriving Eq. (20) the conversion of turbulent energy to acoustic energy was assumed to be complete. This, in reality, cannot be accomplished as evidenced by the fact that turbulence does exist downstream of a combustor. To account for this, an acoustic pressure efficiency $n_{p}$ is introduced and defined as the ratio of the experimentally measured acoustic pressure generated by the combustor $p_{\omega}$ to the acoustic pressure that would be generated if all of the turbulent energy had been converted to an acoustic pressure $P_{\omega}$. From Ref. ()ㅡ $\eta_{p}$ is given by

$$
n_{p}=\frac{p_{\omega}^{\prime}}{p_{\omega}}
$$


Thus the acoustic pressure efficiency can now be determined for any specific combustor. The value of np will probably depend on the combustor type and configuration. Therefore it is necessary that. np be determined for a number of combustors so that the proper value $c$ an be selected when making predictions of combustor pressure fluctuations.

\section{OVERALL SOUND PRESSURE LEVEL}

An expression for the overall sound pressure level can be obtained by multiplying Eq. (20) by the acoustic pressure efficiency $\eta_{p}$, squaring and then integrating over the frequency range of interest. The resulting equation for $P_{\omega}$ is

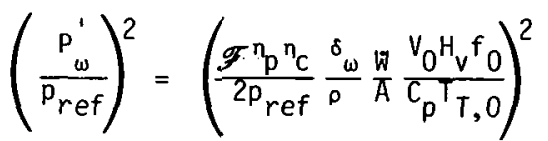

where the term $\mathscr{F}$ contains all of the frequency dependent term and is defined as

$$
\begin{aligned}
\mathscr{F}^{2} \equiv \int_{f_{L}}^{f_{U}} \frac{\lambda}{k}^{2} \frac{1}{\left[1+(k / \lambda)^{2}\right]} d f \\
=\left.\frac{\lambda C}{2 \pi}^{2}\left[\frac{1}{f}+\frac{2 \pi}{\lambda C} \tan ^{-1}\left(\frac{2 \pi f}{\lambda C}\right)\right]\right|_{f_{U}} ^{f_{L}}
\end{aligned}
$$

Equation (23) indicates that $\mathscr{F}^{2}$ is inversely proportional to the frequency. Therefore the magnitude of will be determined by the lower limit on frequency, providing that the upper limit is much greater than the lower limit. In determining the OASPL the combustion noise frequency limits are of the order of 50 and $2000 \mathrm{~Hz}$. For a fuel mass decay constant $\lambda$, defined as $2 \pi / L_{b}$, and reasonable sonic velocities, Eq. (23) can be approximated by using the lower frequency limit by

$$
\mathscr{F}^{2}=\frac{c^{2}}{L_{b}^{2} f}, \sec ^{-1}
$$

The overall sound pressure level (OASPL) equation can now be written for the combustor from Eqs. (22) and (24) as

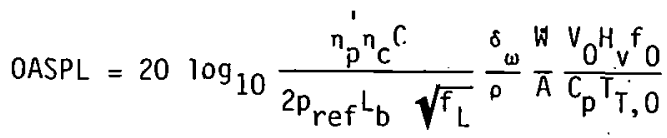

The acoustic pressure efficiency $n_{j}$ can be determined from the measured overall sound pressure level and $\mathrm{Eq}$. (25). The equation for experimentally determining the value of $n_{p}$ is

$$
\eta_{p}^{\prime}=\frac{2 p_{r e f} L_{L} \sqrt{f_{L}}}{n_{c}{ }^{C}} \frac{\rho}{\delta_{\omega}} \underset{W}{A} \frac{C_{p} T_{T}, 0}{V_{0} H_{v}^{f} 0} \times 10^{0 A S P L / 20}
$$

Where the local static temperature is used in calculating the sonic velocity, $C$.

The fluctuating pressure in the combustor of the CF6-50 turbofan engine has been measured $(10-11)$. The measured overall fluctuating pressure (assumed to be acoustic) in the combustor at 3.8 percent of design thrust has been used in conjunction with Eq. (26) to calculate the acoustic pressure efficiency, $n_{p}=$ 0.030 , for the CF6-50 combustor.

From this acoustic pressure efficiency the overall sound pressure level in the combustor was predicted over the engine thrust range by using Eq. (25). The results are shown in Fig. 2. The theory, given by the solid line, agrees well with the measured fluctuating pressure, given by the symbols.

In light of this discussion it can be concluded that the theory predicts the trends in the combustor overall acoustic pressure with engine operating conditions and, for CF6-50 combustors and similar geometries, an acoustic pressure efficiency of $0.030 \mathrm{can}$ be used in Eq. (25) to predict the combustor overall acoustic pressure. The acoustic pressure efficiency has also been determined for a YF102 engine combustor. This combustor, unlike the CF6-50, is a reverse-flow combustor and is much smaller than the CF6-50. Its acoustic pressure efficiency is 0.016 at 30 percent of design speed. Using the acoustic pressure eff $i$ ciency of 0.016 yields only a $0.2-d B$ error in predicting the OASPL at the 95-percent speed. Comparing the acoustic pressure efficiencies shows that the CF6-50 combustor converts the turbulence to acoustic pressure more efficiently than the YF102 combustor.

\section{NARROW-BAND SOUND PRESSURE LEVEL SPECTRUM}

The theoretical sound pressure spectrum is given by $\mathrm{Eq} .(20)$. Discarding the phase information contained in the exponential term and applying Eq. (21) to account for the efficiency of conversion of turbulence to acoustic pressure, Eq. (20) is written as

$\frac{2}{n_{p} n_{C}} \frac{\rho}{\delta_{\omega}} \frac{{ }^{A L_{b}}}{W} \frac{C_{p}^{T} T, 0^{p}}{V_{0}{ }^{H} v_{0}{ }^{C}}=\frac{1}{f} \frac{1}{\left[1+\left(f L_{b} / C\right)^{2}\right]^{1 / 2}}$

The right side of Eq. (27) represents the spectral shape of the acoustic pressure given by Eq. (20). The sound pressure level spectrum is given by

$S P L=20 \log _{10} \frac{{ }^{n} c \eta_{p}}{2 p_{\text {ref }}} \frac{\delta}{\rho} \frac{{ }^{\omega}}{A L_{b}} \frac{V_{0} H_{v} f_{0}}{C_{P} T_{T, 0}} c \frac{\Delta f}{f\left[1+\left(f L_{b} / C\right)^{2}\right]^{1 / 2}}$

This equation states that the acoustic pressure level is inversely proportional to the square of the frequency since the term under the radical is small for $\mathrm{fL}_{\mathrm{b}}<\mathrm{C}$.

The predicted spectral shape is compared with the measured narrow-band spectra for the CF6-50 engine (10) in Fig. 3. The measured fluctuating pressure level in the combustor of the CF6-50 turbofan engine operating at 3.8 percent of design thrust is shown in Fig. $3(\mathrm{a})$. Also shown in Fig. $3(\mathrm{a})$ is a plot of Eq. (28) with the level matched to the measured spectrum at a frequency of $2000 \mathrm{~Hz}$. The acoustic pressure efficiency is determined from Eq. (28) and is given by 


$$
\begin{aligned}
& n_{p}=\frac{2}{n_{C}} \frac{\rho}{\delta_{\omega}} \frac{{ }^{A L} L_{b}}{W} \frac{{ }_{p}^{C} T_{,} 0^{p} \cdot r e f}{V_{0} H_{v} f_{0}^{C}} \quad 10^{S P L(f) / 20} \frac{f}{\Delta f} \\
& x\left[1+\left(\frac{f L_{b}}{C}\right)^{2}\right]^{1 / 2}
\end{aligned}
$$

At $2000 \mathrm{~Hz}$ the measured $\mathrm{SPL}$ is equal to $110 \mathrm{~dB}$ and the narrow-band spectral acoustic pressure efficiency, computed by using Eq. (29), is

$$
n_{p}=4.5 \times 10^{-2}
$$

when $\delta_{\omega} / \rho$ is assumed to be 0.3 .

The trends in the spectral shape of both theory and experiment agree very well at the 3.8-percentthrust point given in Fig. 3(a). The comparison at 99.8-percent thrust shown in Fig. 3(b), however, is not as good. The theory given for the 99.8-percentthrust point uses the acoustic pressure efficiency obtained from the 3.8-percent-thrust point, namely $n_{p}=4.5 \times 10^{-2}$. Examining Fig. 3(b) shows that the general trend is correct but that large oscillations in the spectrum are not predicted by Eq. (28). Acoustic reflections from the turbine may account for these oscillations.

From this discussion it is concluded that the spectrum predicted by Eq. (28) agrees substantially with the experimental measurements made in an operating engine. It can also be concluded that the simplified combustion noise theory given herein substantially predicts the trends in the acoustic pressures generated by the combustion process of turbine engine combustors.

\section{1/3-OCTAVE-BAND SOUND PRESSURE SPECTRUM}

The 1/3-octave-band spectrum of the combustor noise can be calculated for a simplified spectrum by neglecting the term under the radical contained in Eq. (28) and integrating over the $1 / 3$ octave bandwidth:

$$
\Delta S P L=10 \log _{10} \frac{0.23}{f_{c}}
$$

This equation has been plotted in Fig. 4 with its level matched, as for the narrow-band spectrum, at the 2000-Hz band for the CF6-50 operating at the 3.8percent-thrust point. The 1/3-octave-band data given by the symbols match the theoretical trends for frequencies above $100 \mathrm{~Hz}$. At frequencies less than $100 \mathrm{~Hz}$ the data are significantly over predicted by the theory; a result attributable to the absence of low frequency turbulence.

\section{EARTH TO ORBIT PROPULSION SYSTEM PRESSURE SPECTRUM}

Turbopump systems used in earth to orbit propulsion systems are subjected to fluctuating pressures that could cause a decrease in their life expectancy due to high cycle loading of structures. Equation (28) was used to predict the fluctuating pressures in the ducting downstream of the hydrogen pump preburner using, as a first approximation, the CF6-50 turbofan acoustic pressure efficiency reported herein. Reference (5) gives the geometry of the hydrogen pump preburner, turbine, and ducting leading to the main engine dome. Figure 5 compares the predicted pressure spectrum in the preburner to the measured pressure downstream of the turbine. The turbine will attenuate some of the preburner generated acoustic signal but it does not decrease the theoretically predicted value enough to cause serious disagreement.

\section{CONCLUS IONS}

By using the theory developed herein, expressions for the acoustic pressure generated by the combustion process have been derived. It has been shown that the overall acoustic pressure in large turbofan engine combustors can be predicted over the range of engine operating speeds, providing that the acoustic pressure efficiency, defined herein, can be determined. The following conclusions are based on the theory:

1. The major source of combustor pressure fluctuations is the interaction of the turbulence and the mean internal energy (i.e., heat) additions in the combustor.

2. For a typical large turbofan engine the ratio of the measured overall acoustic pressure in the combustor to the theoretical acoustic pressure (obtained by assuming that all of the turbulent energy is converted to acoustic pressure) is a constant with a magnitude of the order of 0.030 . For smaller reverseflow combustors the acoustic pressure efficiency has been found to be of the order of 0.020 .

3. The turbulence-flame-generated noise is directly proportional to the square of the sonic velocity, the mass flow rate per unit cross-sectional area, the ratio of heat energy added per unit mass of air to the inlet enthalpy, the inlet velocity, the combustion efficiency, and the turbulence intensity. It is inversely proportional to the square of the frequency and the burning length of the combustor. 4. Because combustor size limits turbulence scales, larger scale low frequency turbulence is suppressed.

\section{APPENDIX A}

Symbols
A

C

$C_{p}$

$\mathrm{c}_{0}$

$c_{1}, c_{2}$

f

$f_{L}$

$f_{U}$

$f_{0}$

$\Delta f$

$g$

$\mathrm{H}_{\mathrm{T}}$

$\mathrm{H}_{\mathrm{v}}$ i 


$\begin{array}{ll}J & \text { mechanical equivalent of heat, } J / \mathrm{kg} \mathrm{m} \\ \mathrm{k} & \text { acoustic wave number, } \omega / \mathrm{C}_{0}, 1 / \mathrm{m} . \\ \mathrm{L}_{\mathrm{b}} & \text { burning length (i.e., axial distance } \\ & \text { measured from } \mathrm{flame} \text { front to point in } \\ & \text { combustor where chemical reaction ends), } \mathrm{m} \\ \mathrm{L}_{T} & \text { length of turbulence generating region, } \mathrm{m} \\ \mathrm{M} & \text { Mach number } \\ \mathrm{SPL} & \text { sound pressure level, dB (re } 20 \mu \mathrm{Pa}) \\ \text { OASPL } & \text { overall sound pressure level, dB (re } 20 \mu \mathrm{Pa}) \\ \mathrm{P} & \text { instantaneous or mean static pressure, } \mathrm{Pa} \\ \mathrm{p} & \text { time-fluctuating part of instantaneous } \\ & \text { pressure, Pa } \\ \mathrm{P}_{\mathrm{ref}} & \text { reference pressure used in calculating sound } \\ \mathrm{p}_{\omega} & \text { Fressure level, } 20 \mu \mathrm{Pa} \\ & \text { Fourier-transformed fluctuating pressure, } \\ & \text { acoustic pressure generated in combustor, } \\ & \text { Pa sec }\end{array}$

$P_{\omega, L} \quad$ acoustic pressure for left-running wave (i.e., $x<0)$, Pa sec

$P_{\omega, R} \quad$ acoustic pressure for right-running wave

(i.e., $x>L_{b}$ ), Pa sec

$Q$ heat energy, $\mathrm{J} / \mathrm{kg}(\mathrm{cal} / \mathrm{kg})$

$R$ universal gas constant for $a i r, m / K$

S' fluctuating component of entropy, J/kg K

$T$ mean temperature, $K$

$T^{\prime} \quad$ instantaneous or fluctuating temperature, $K$

$t$ time, sec

$U$ instantaneous or mean internal energy, $\mathrm{J} / \mathrm{kg}$

$v \quad$ mean velocity, $\mathrm{m} / \mathrm{sec}$

$v \quad$ instantaneous or fluctuating velocity, $\mathrm{m} / \mathrm{sec}$

$W \quad$ mean mass flow rate, $\mathrm{kg} / \mathrm{sec}$

$x_{b}$ axial distance measured from flame front, positive in direction of flow, $m$

$x_{T}$ distance measured from turbulence generator inlet, $m$

$\triangle S P L \quad$ sound pressure level difference

$\delta$ time-fluctuating component of density, $\mathrm{kg} / \mathrm{m}^{3}$

$n_{c} \quad$ combustion efficiency

$n_{p} \quad$ acoustic pressure efficiency

$\theta \quad$ phase angle, deg

$\lambda$ fuel mass decay constant, assigned a value of $2 \pi / L_{b}$

$\xi$ axial distance in combustor measured from

flame front location, $m$

p instantaneous or mean density of fluid, $\mathrm{kg} / \mathrm{m}^{3}$

$\varphi_{\omega} \quad$ Fourier-transformed source terms (Eq. (8))

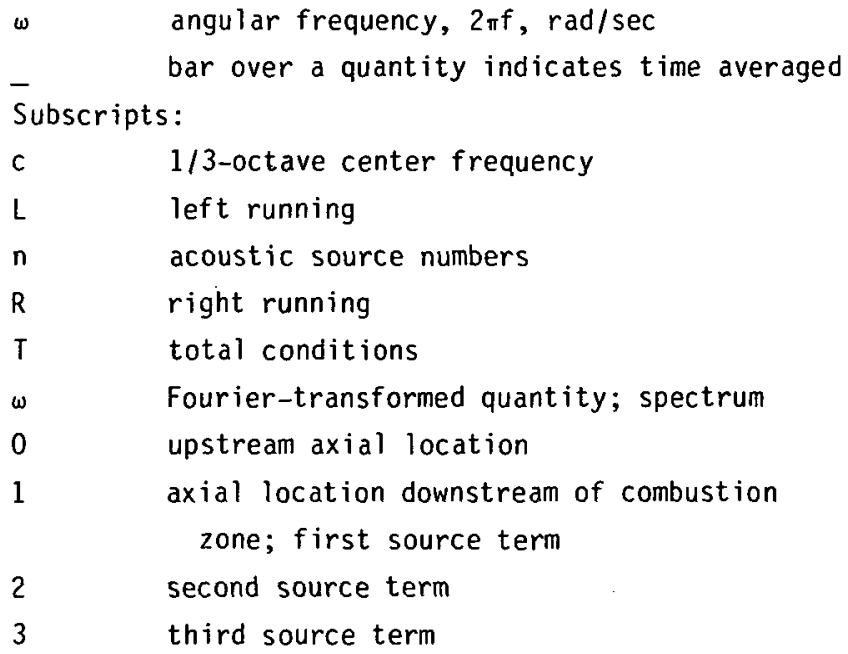

\section{APPENDIX B}

Acoustic Source Term Derivation are:

The acoustic source terms as defined in Ref. (ㅁ)

$$
\begin{aligned}
& \varphi_{1} \equiv \frac{\partial^{2}}{\partial t^{2}}\left(\frac{p}{c_{0}^{2}}-\delta\right) \\
& \varphi_{2} \equiv 2 \frac{\partial^{2}(\rho V v)}{\partial x^{2}} \\
& \varphi_{3} \equiv \frac{\partial^{2}\left(\delta v^{2}\right)}{\partial x^{2}}
\end{aligned}
$$

For ease in solving the acoustic wave equation for the particular integral and because information concerning the acoustic pressure is usually presented as a function of frequency the Fourier transforms of the source terms are required, they are:

$$
\begin{aligned}
& \varphi_{1, \omega}=-\omega^{2}\left(\frac{p_{\omega}}{C_{0}^{2}}-\delta_{\omega}\right) \\
& \varphi_{2, \omega}=2 \frac{\partial^{2} \rho V v_{\omega}}{\partial x^{2}} \\
& \varphi_{\omega, 3}=\frac{\partial^{2} \delta v^{2}}{\partial x^{2}}
\end{aligned}
$$

The first source term is related to the fluctuating heat release in the combustion zone. From the first law of thermodynamics in differential form:

$$
d Q=d U-\frac{P d \rho}{J_{\rho}^{2}}
$$


dividing by the temperature and specifying a calorically and thermally perfect gas yields this expression in terms of entropy as

$$
\begin{aligned}
& \frac{d S}{C_{v}}=\frac{d T}{T}-\frac{R}{d C_{v}} \frac{d \rho}{\rho} \\
& \frac{d S}{C_{v}}=\frac{d T}{T}-(\gamma-1) \frac{d_{\rho}}{\rho}
\end{aligned}
$$

For the thermally perfect gas

$$
\frac{d T}{T}=\frac{d P}{P}-\frac{d \rho}{\rho}
$$

Substituting Eq. (B10) into (B9) yields

$$
\rho \frac{d S}{C p}=\frac{\rho d P}{\gamma P}-d \rho
$$

For the mean sonic velocity given by

$$
C_{0}=\overline{r R T}
$$

Equation (B11) maybe written as:

$$
\frac{\rho S^{\prime}}{C_{p}}=\frac{p}{C_{0}^{2}}-\delta
$$

The first source term Eq. (B4) may, by substituting Eq. (B13), be written in terms of the fluctuating entropy as:

$$
\begin{aligned}
& \varphi_{1, \omega}=-\omega^{2} \frac{\rho S_{\omega}^{\prime}}{C_{p}} \\
& \text { For } d Q \equiv d\left(H_{v} f_{0}\right) \\
& S^{\prime}=\frac{H_{v} f_{0}^{\prime}}{T}
\end{aligned}
$$

and Eq. (B14) becomes

$$
\varphi_{1, \omega}=-\frac{\omega^{2}{ }^{2} H_{v} f_{0, \omega}}{C_{p} T}
$$

The only term in Eq. (B16) that is fluctuating with time is the fuel-air ratio. Hence this source term describes the contribution of the fluctuating fuel-air ratio to the acoustic pressure.

The second source term, $92, w, E q$. (B5) may be written for steady mean flow'as:

$$
\varphi_{2, \omega}=2 \frac{\omega}{A} \frac{\partial^{2} v_{\omega}}{\partial x^{2}}
$$

This acoustic source contains the turbulent velocities generated by either turbulators in air breathing turbine engine combustors or by the mixing of the propellants in rocket engine combustion chambers. To represent the turbulence source generation the turbulent velocity will be assumed to be an exponential function of distance given by:

$$
v_{\omega}=v_{\omega, L}\left[1-e^{-\lambda} \frac{x_{T}}{L_{T}}\right]
$$

where $X_{T}$ is measured from the upstream side of the turbulator and $L_{T}$ is defined as the axial distance measured from the front of the turbulence generator to the point where the turbulent velocity $v_{\omega}\left(L_{T}\right)$ reaches its maximum. The value of $\lambda$ is taken as $2 \pi$ so that at $X=L T$, the turbulent velocity $v$ reaches the maximum. Taking the Laplacian of $v_{\omega}$

$$
\frac{\partial^{2} v_{\omega}}{\partial x^{2}}=-v_{\omega, L}\left(\frac{\lambda}{L_{T}}\right)^{2} e^{-\lambda x_{T}}
$$

Substituting Eq. (B19) into Eq. (B17) gives:

$$
\varphi_{2, \omega}=-2 \frac{W}{A} v_{\omega, L}\left(\frac{\lambda}{L_{T}}\right)^{2} e^{-\lambda x_{T}}
$$

The third source term $\$ 3, w$ Eq. (B6) has been investigated in Ref. (6). "An exponential decay of the fuel droplet with distance from the flame front was assumed due to mixing and burning of the fuel droplet. Steady flow was assumed and the source term written for low Mach number flow as:

$$
\Phi_{3, \omega}=-\frac{\omega}{A} \frac{\delta}{\rho} v_{0} \frac{{ }_{C_{C}{ }^{H} v^{f} f_{0}{ }^{2}}}{C_{p}{ }^{\top} T, 0} e^{-\lambda X}
$$

\section{REFERENCES}

1. Huff, R. G., Clark, B. J., and Dorsch, R. G., "Interim Prediction Method for Low Frequency Core Engine Noise," NASA TM X-71627, 1974.

2. Mathews, D. C. and Peracchio, A. A., "Progress in Core Engine and Turbine Noise Technology," AIAA Paper No. 74-948, Aug. 1974.

3. Reshotko, M. and Karchmer, A., "Core Noise Measurements from a Small, General Aviation Turbof an Engine," NASA TM-81610, 1980.

4. Huff, R. G., Groesbeck, D. E., and Goodykoontz, J. H., "Low Frequency Noise in a Quiet, Clean, General Aviation Turbofan Engine," NASA TM-83520, Oct. 1983.

5. Liang, P. Y., "An Inviscid Three-Dimensional Analysis of the Space Shuttle Main Engine Hot-Gas Manifold," AIAA Paper No. 83-1523, June 1983.

6. Huff, R. G., "A Simplified Combustion Noise Theory Yielding a Prediction of Fluctuating Pressure Level," NASA TP-2237, 1983.

7. Bird, R. B., Stewart, W. E., and Lightfoot, E. N., Transport Phenomena, Wiley, New York, 1960, pp. 322-323. 
8. Wylie, C. R., Jr., Advanced Engineering Mathematics, 2nd ed., McGraw-Hill, New York, 1960.

9. Hildebrand, F. B., Methods of Applied Mathematics, Prentice-Hall, New York, 1952 .

10. Doyle, V. L., "Core Noise Investigation of the CF6-50 Turbof an Engine, Data Report," R79AEG247, General Electric Co., Aircraft Engine Group, Cincinnati, Ohio, Jan. 1980. (NASA CR-159598.)
11. Doyle, V. L., and Moore, M. T., "Core Noise Investigation of the CF6-50 Turbofan Engine, Final Report," R79AEG395, General Electric Co., Aircraft Engine Group, Cincinnati, Ohio, Jan. 1980. (NASA CR-159749.) 


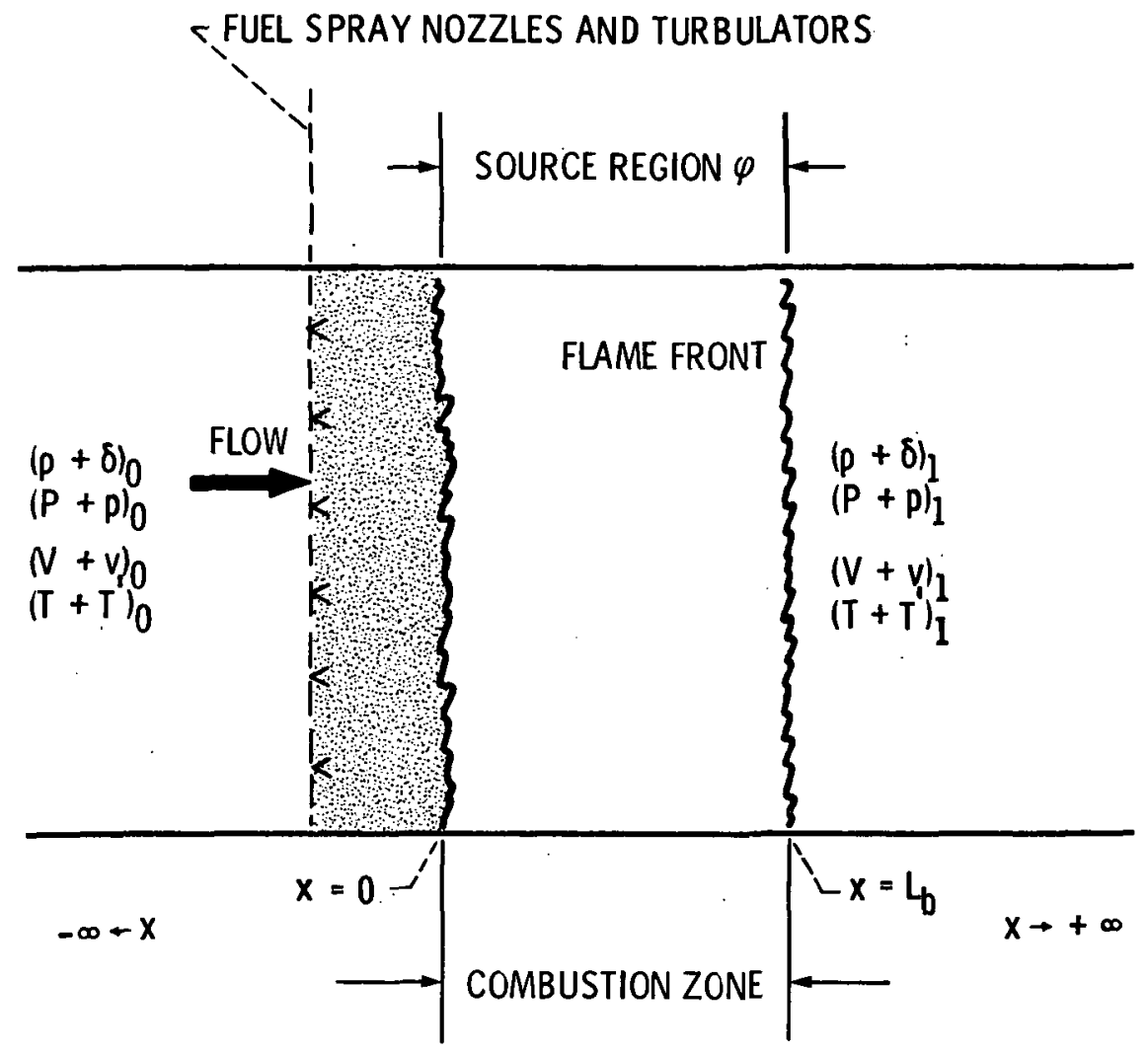

Figure 1. - Combustion noise source model.

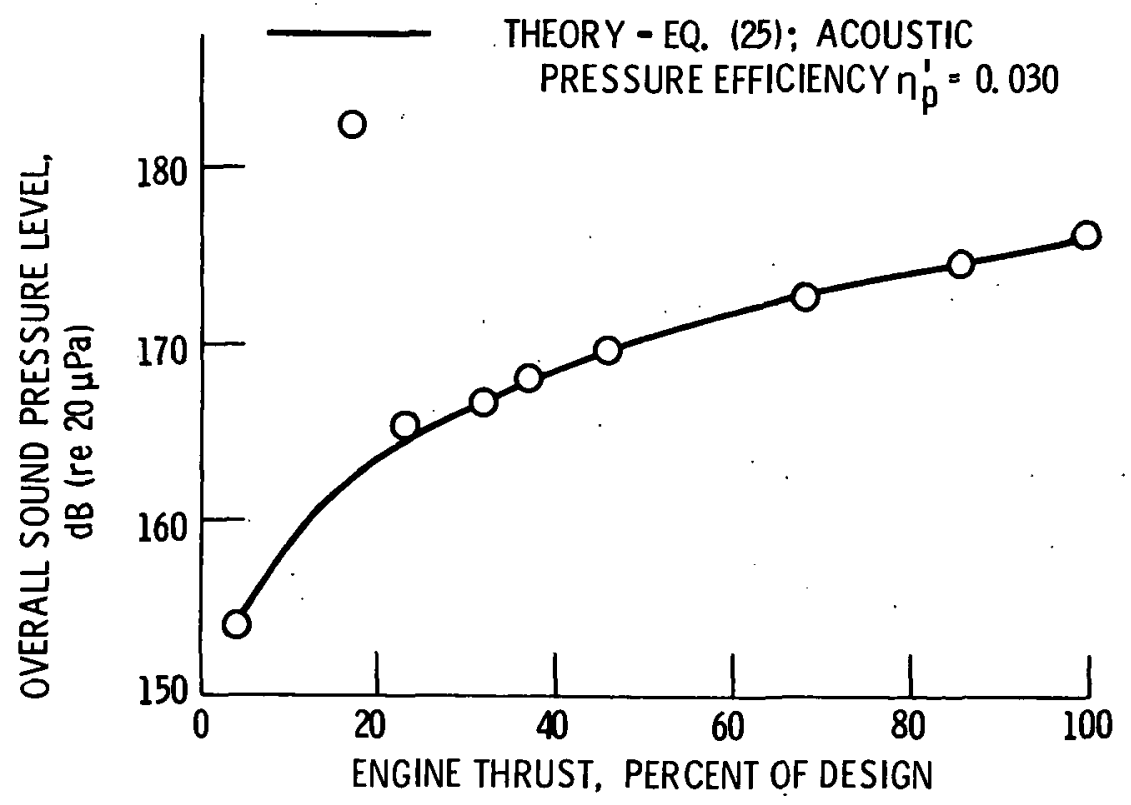

Figure 2. - Combustor pressure fluctuations as a function of CF6-50 turbofan engine thrust-experimental data and theory. 


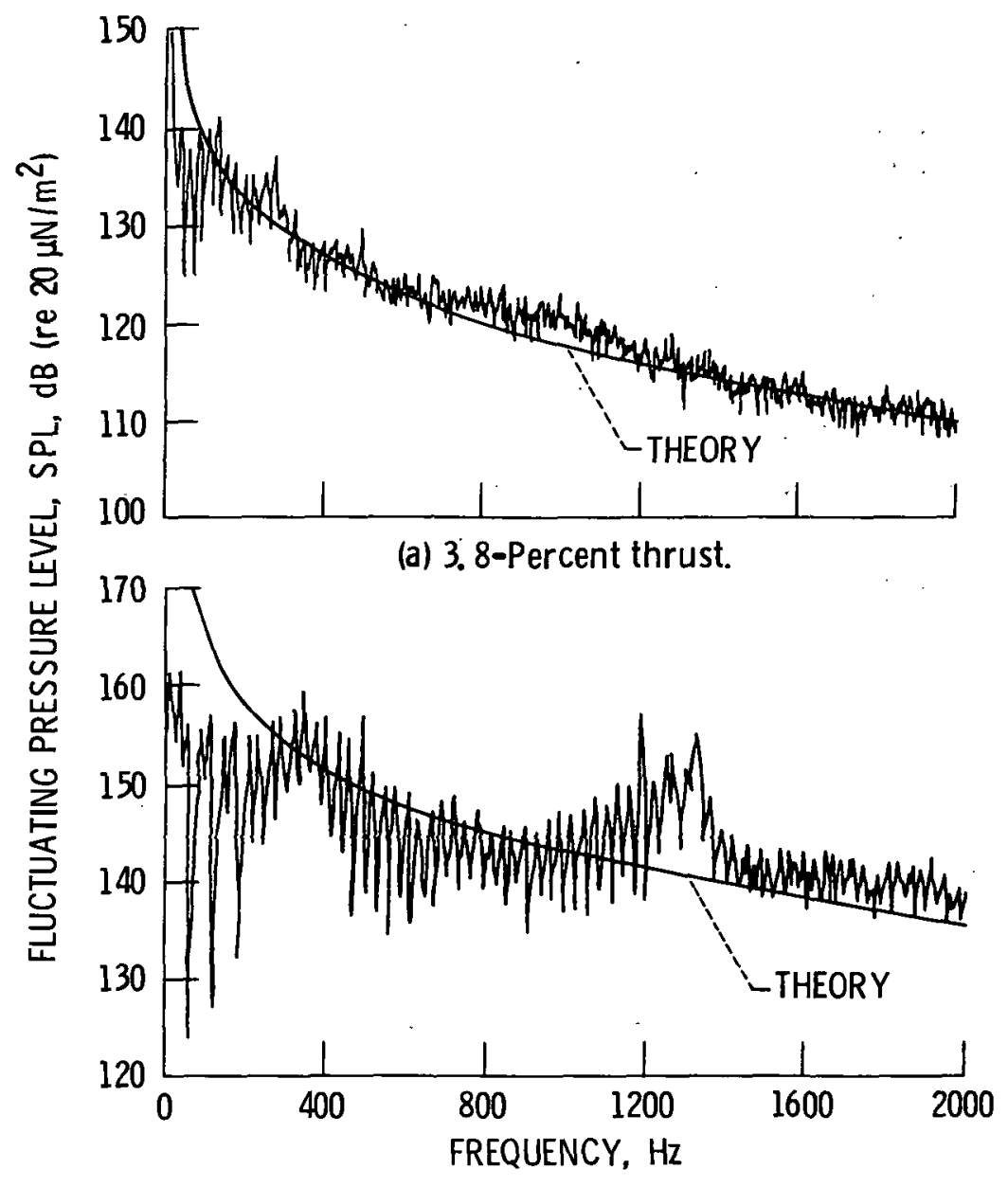

(b) 99.8-Percent thrust.

Figure 3. - Comparison of theory to measured sound pressure level spectrum-CF6-50 turbofan engine combustor. Acoustic pressure efficiency, $\eta_{p}$, 4. $5 \times 10^{-2}$; dimensionless fluctuating density, $\delta_{\omega} / p, 0,3$. 


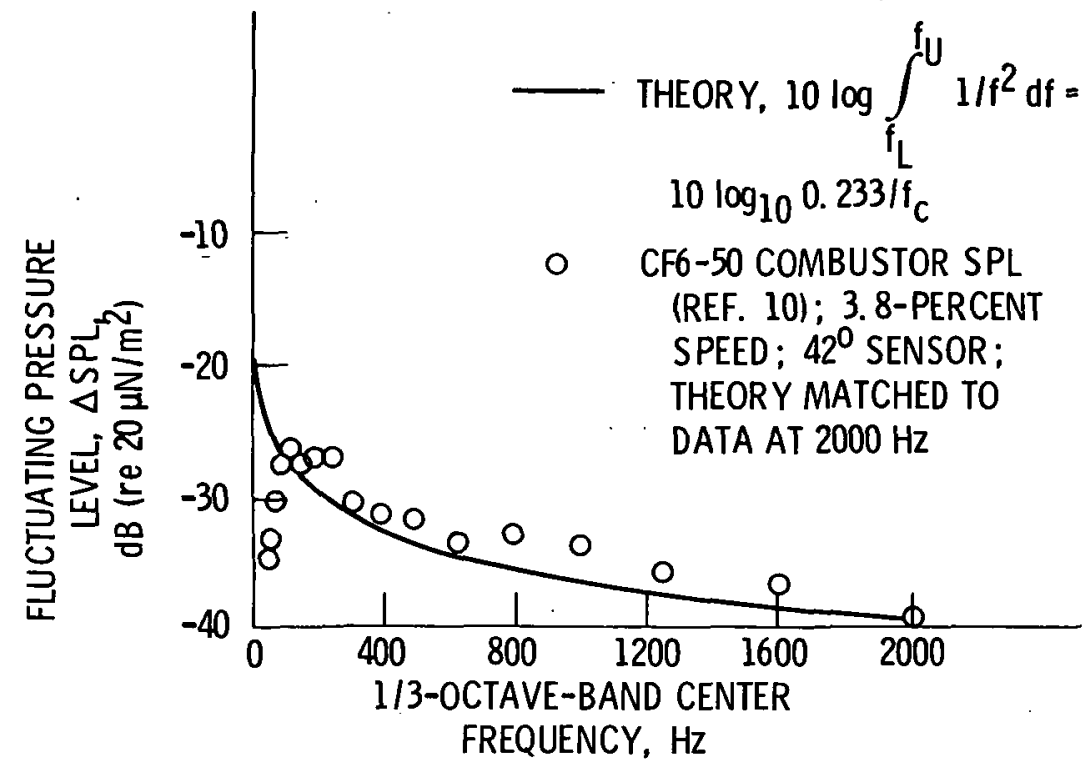

Figure 4. - 1/3-0ctave-band fluctuating pressure level spectrum shape for $\mathrm{CF} 6-50$ turbofan engine combustor. Thrust, 3.8 percent of design.

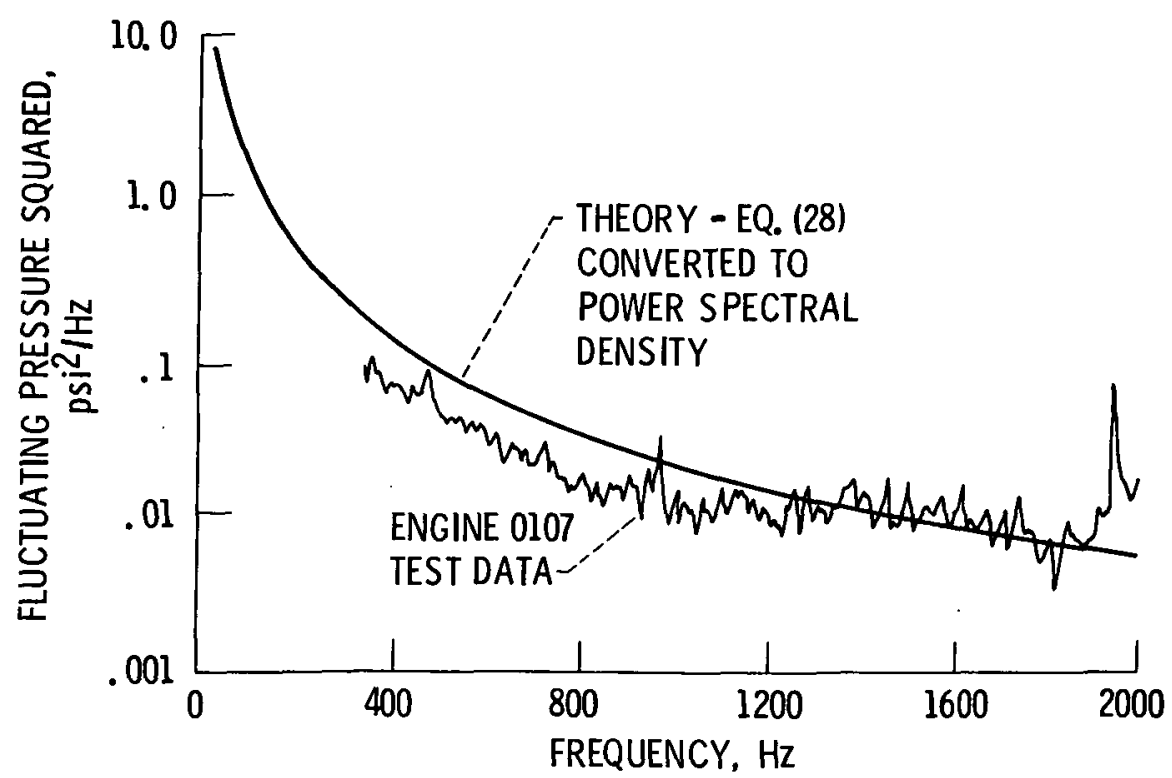

Figure 5. - Comparison of the theoretically predicted earth to orbit propulsion systems hydrogen pump preburner fluctuating pressure spectrum to the measured spectrum downstream of the turbine. 


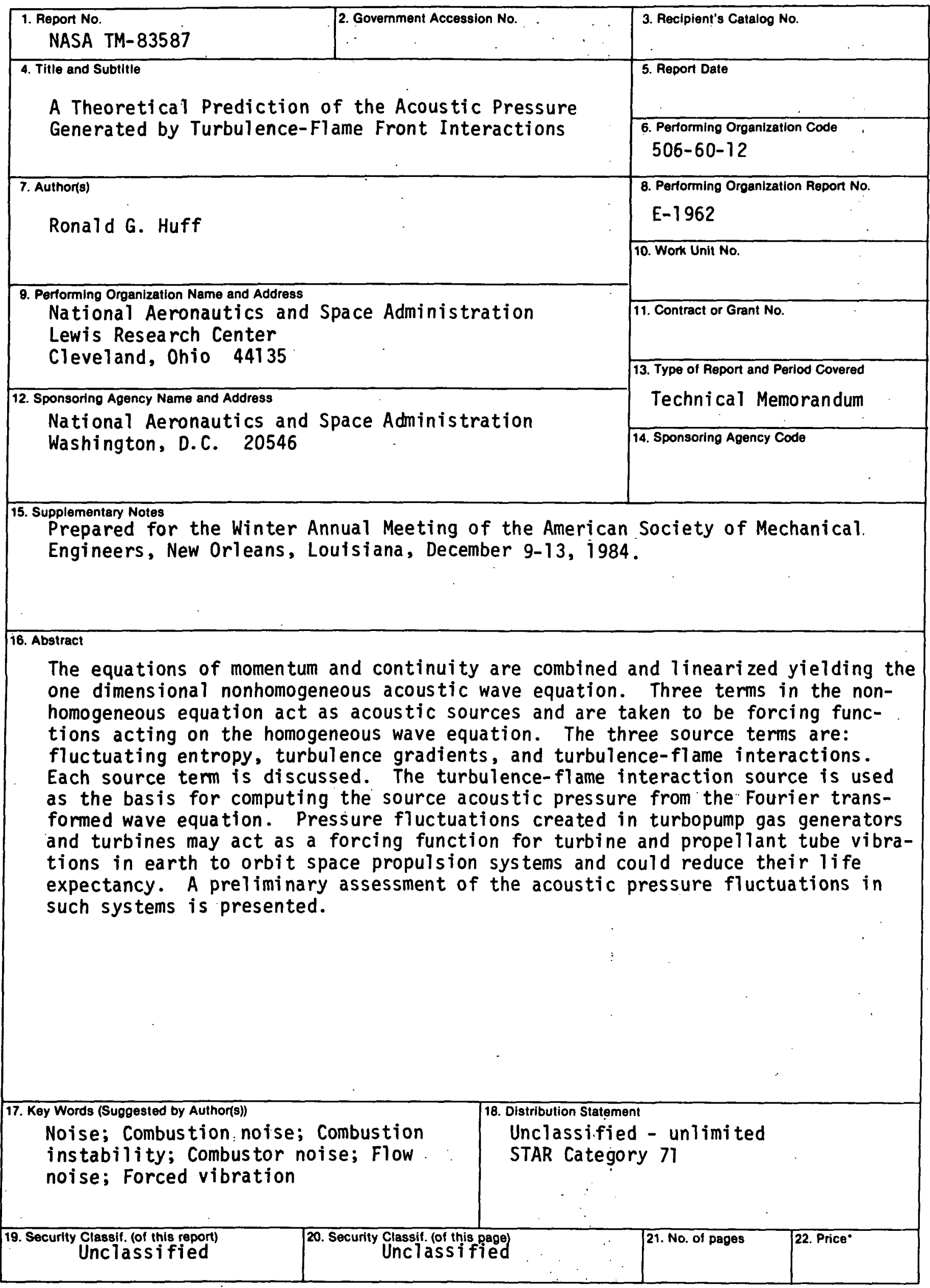

"For sale by the National Technical Information Service, Springfield, Virginia 22161 
National Aeronautics and Space Administration

Washington, D.C.

20546

Official Business

Penalty for Private Use, $\$ 300$
SPECIAL FOURTH CLASS MAIL BOOK

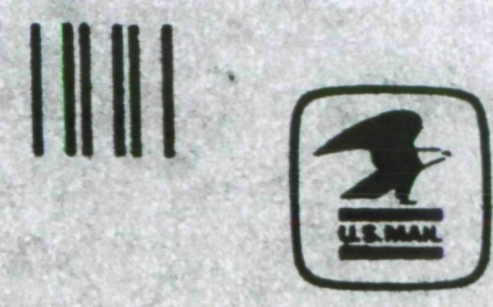

Poetege and Faes Paid National Aeroneutics and Spece Administration NASA-451 